\title{
Toxoplasmosis congénita en gemelos
}

\author{
Congenital toxoplasmosis in twins: case report
}

\author{
Martín Brizuela', Eduardo Otero², Andrea Molina², Karina De Risio ${ }^{2}$, Jimena Mariano ${ }^{3}$ y Gustavo Cañete ${ }^{1}$
}

'Servicio de Infectología. Hospital Zonal General de Agudos "Dr. Isidoro G Iriarte"- Quilmes. Provincia de Buenos Aires, Argentina.
'Servicio de Neonatología. Hospital Zonal General de Agudos "Dr. Isidoro G Iriarte"- Quilmes. Provincia de Buenos Aires, Argentina.
${ }^{3}$ Servicio de Oftalmología Hospital Zonal General de Agudos "Dr. Isidoro G Iriarte"- Quilmes. Provincia de Buenos Aires, Argentina.

Financiación externa: ninguna

Conflicto de intereses: ninguno que declarar

Recibido: 4 de octubre de 2019 / Aceptado: 7 de junio de 2020

\section{Resumen}

La toxoplasmosis congénita en embarazos gemelares es infrecuente. Presentamos el caso de un embarazo gemelar monocigótico, monocorial con una infección materna por toxoplasmosis diagnosticada a las 33 semanas de gestación mediante detección de IgM e IgG reactivas y test de baja avidez de IgG. No pudo realizarse la detección del parásito en líquido amniótico debido a que no se hizo amniocentesis. Las recién nacidas $(\mathrm{RN})$ de término presentaron calcificaciones cerebrales y coriorretinitis activa bilateral, sin otras manifestaciones clínicas asociadas. Tuvieron IgG e IgM reactiva para toxoplasmosis. No se realizó RPC para Toxoplasma gondii en placenta ni en sangre de cordón. Recibieron tratamiento durante el primer año de vida con pirimetamina, sulfadiazina y ácido folínico, sin eventos adversos asociados a la terapia. La metodología diagnóstica y el tratamiento no difieren de los embarazos con feto único. Uno o ambos RN pueden estar comprometidos. Es fundamental el seguimiento multidisciplinario para la detección precoz de reactivaciones o progresión de las lesiones.

Palabras clave: toxoplasmosis; Toxoplasma gondii; embarazo; gemelas.

\section{Introducción}

L a toxoplasmosis es una infección parasitaria de distribución mundial producida por el protozoo Toxoplasma gondii. La infección fetal se produce por la transmisión hematógena del parásito a través de la placenta durante la primoinfección materna ${ }^{1}$. $\mathrm{Su}$ incidencia durante el embarazo varía desde 0,5-3 casos/1.000 embarazos susceptibles en Europa hasta 4,8-5,7 casos/1.000 embarazos

\begin{abstract}
Congenital toxoplasmosis in twin pregnancies is infrequent. We present the case of a monozygotic and monocorial twin pregnancy with maternal toxoplasmosis infection diagnosed at 33 weeks gestation by detecting reactive IgM and IgG and low avidity test for IgG. The detection of the parasite in amniotic fluid could not be performed because the amniocentesis was not done. The term newborns presented cerebral calcifications and bilateral active chorioretinitis without other associated clinical manifestations. They had IgG and IgM reactive for toxoplasmosis. The detection of Toxoplasma gondii was not performed by placental PCR or cord blood. They received treatment during the first year of life with pyrimethamine, sulfadiazine and folinic acid. They had no adverse events associated with the therapy. The diagnostic assessment and treatment does not differ from pregnancies with a single fetus. One or both newborns may be compromised. Multidisciplinary monitoring is essential for the early detection of reactivations or progression of lesions.
\end{abstract}

Keywords: toxoplasmosis; Toxoplasma gondii; pregnancy; twins.

susceptibles en Latinoamérica ${ }^{1}$. La media de incidencia global es de 1,5/1.000 recién nacidos (RN) vivos ${ }^{2,3}$. En Argentina se estima una incidencia de 2,5- 3,5 casos/1.000 RN vivos.

La infección congénita por $T$. gondii en embarazos gemelares es un hecho poco frecuente. Hasta el momento, los datos se basan en reportes o series de $\operatorname{casos}^{4-6}$.

Presentamos los casos clínicos de dos gemelas con una infección congénita por toxoplasmosis con compromiso cerebral y ocular. 
por RPC (reacción de polimerasa en cadena) en líquido amniótico.

Nacieron gemelas de sexo femenino de 38 semanas de edad gestacional (Tabla 1). El examen físico al nacer fue normal. En el fondo de ojos ambas presentaron signos de coriorretinitis activa bilateral (Figura 1) y en las ecografías y TC cerebrales se detectaron calcificaciones cerebrales (Figura 2A y 2B). No se realizó RPC en sangre ni en líquido cefalorraquídeo (LCR) para toxoplasmosis. No se estudió la placenta ni la sangre de cordón umbilical. No se realizó punción lumbar para obtención de LCR debido a que el compromiso neurológico fue confirmado por la presencia de lesiones oculares y cerebrales. En base a los antecedentes maternos, resultados serológicos de ambas gemelas y hallazgos compatibles en el fondo de ojos e imágenes cerebrales, y habiéndose descartado otras infecciones que pueden producir un cuadro similar, se hizo el diagnóstico de toxoplasmosis congénita sintomática con compromiso neurológico.

\begin{tabular}{|c|c|c|}
\hline Variable & Gemela 1 & Gemela 2 \\
\hline Edad gestacional (sem)/peso (gramos) & $38 / 2.160$ & $38 / 2.060$ \\
\hline Laboratorio & $\begin{array}{l}\text { FAL: } 192 \mathrm{UI} / \mathrm{mL} \\
\text { GOT: } 56 \mathrm{UI} / \mathrm{mL} \\
\text { GPT: } 11 \mathrm{UI} / \mathrm{mL} \\
\text { BT: } 2,99 \mathrm{mg} / \mathrm{dL} \\
\text { BD: } 0,26 \mathrm{mg} / \mathrm{dL} \\
\text { Glóbulos blancos } 7.500 / \mathrm{mm}^{3} \text { ( } 28 \% \text { neutrófilos, } 49 \% \text { linfocitos, } \\
16 \% \text { monocitos) } \\
\text { Hemoglobina } 18,2 \mathrm{mg} / \mathrm{dL} \text { Plaquetas } 213.000 / \mathrm{mm}^{3}\end{array}$ & $\begin{array}{l}\text { FAL: } 685 \mathrm{UI} / \mathrm{mL} \\
\text { GOT: } 42 \mathrm{UI} / \mathrm{mL} \\
\text { GPT: } 19 \mathrm{UI} / \mathrm{mL} \\
\text { BT: } 6,21 \mathrm{mg} / \mathrm{dL} \\
\text { BD: } 0,71 \mathrm{mg} / \mathrm{dL} \\
\text { Glóbulos blancos } 8.630 / \mathrm{mm}^{3}(30 \% \text { neutrófilos, } 52 \% \text { linfocitos, } \\
12 \% \text { monocitos) } \\
\text { Hemoglobina } 15,8 \mathrm{mg} / \mathrm{dL} \text { Plaquetas } 168.000 / \mathrm{mm}^{3}\end{array}$ \\
\hline Serologías & $\begin{array}{l}\text { IgM toxoplasmosis índice } 4,5 \\
\text { IgG toxoplasmosis } 315 \mathrm{UI} / \mathrm{mL} \\
\text { VDRL no reactiva } \\
\text { IgM CMV no reactiva } \\
\text { IgG CMV no reactiva }\end{array}$ & $\begin{array}{l}\text { IgM toxoplasmosis índice } 3,8 \\
\text { IgG toxoplasmosis } 490 \mathrm{UI} / \mathrm{mL} \\
\text { VDRL no reactiva } \\
\text { IgM CMV no reactiva } \\
\text { IgG CMV no reactiva }\end{array}$ \\
\hline Fondo de ojos & $\begin{array}{l}\text { Focos de coriorretinitis, con turbidez de vítreo y sin presencia } \\
\text { de sinequias }\end{array}$ & $\begin{array}{l}\text { Ojo derecho: cristalino transparente con mínima vitreítis. Foco } \\
\text { de coriorretinitis activa con pliegue de retina } \\
\text { Ojo izquierdo: mala dilatación por sinequias, vitreítis, foco } \\
\text { periférico con pliegue menor. }\end{array}$ \\
\hline Ecografía cerebral & $\begin{array}{l}\text { Línea media conservada, aumento de la ecogenicidad del } \\
\text { parénquima cerebral con visualización de calcificaciones en el } \\
\text { mismo. Redondeamiento de astas frontales y calcificaciones. } \\
\text { Cuerpo calloso presente. Dimensiones de ventrículo izquierdo } \\
\text { (VI) asta frontal (AF) } 0,29 \text { y asta occipital (AO) } 1,99 \text {, ventrículo } \\
\text { derecho (VD) AF 0,35 y AO } 2\end{array}$ & $\begin{array}{l}\text { Línea media centrada. Sistema ventricular conservado. Imáge- } \\
\text { nes compatibles con calcificaciones en parénquima cerebral. } \\
\text { Tronco y fosa posterior normales. Cuerpo calloso presente }\end{array}$ \\
\hline Ecografía abdominal & Normal & Normal \\
\hline Otoemisiones acústicas & Normales & Normales \\
\hline
\end{tabular}




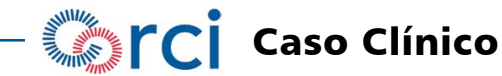

Figura 1. A: fondo de ojos con focos de coriorretinitis en región macular y vitreítis (flecha). B: fondo de ojos con signos de cicatrización de la lesión inicial (flecha).

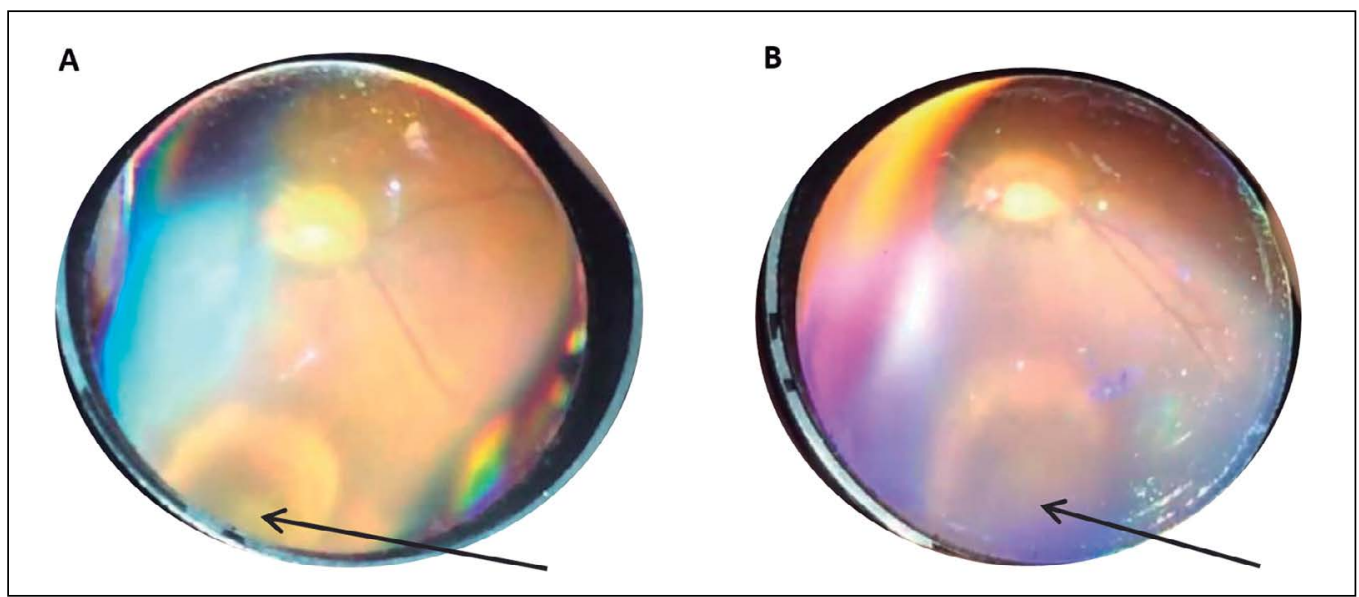

A
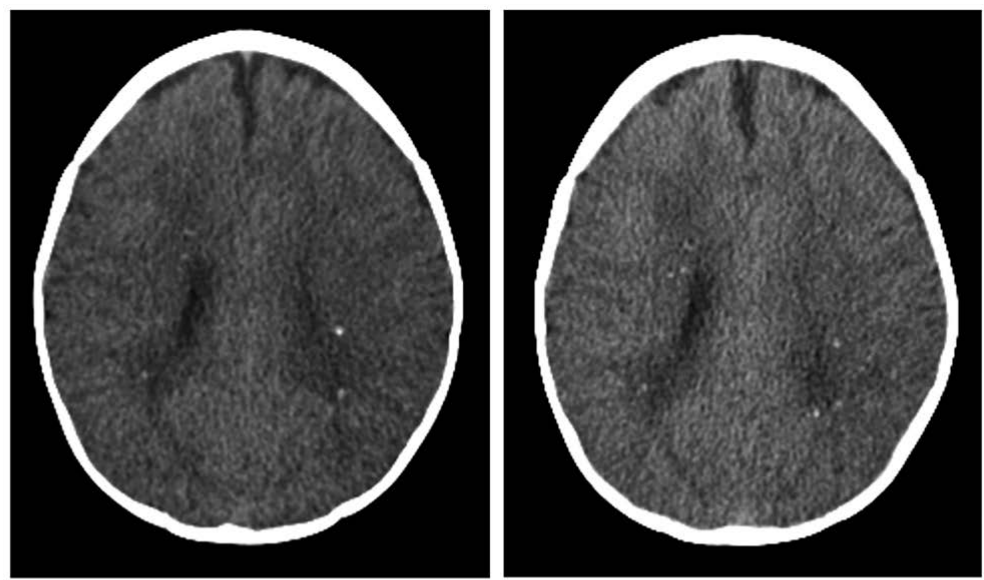

B
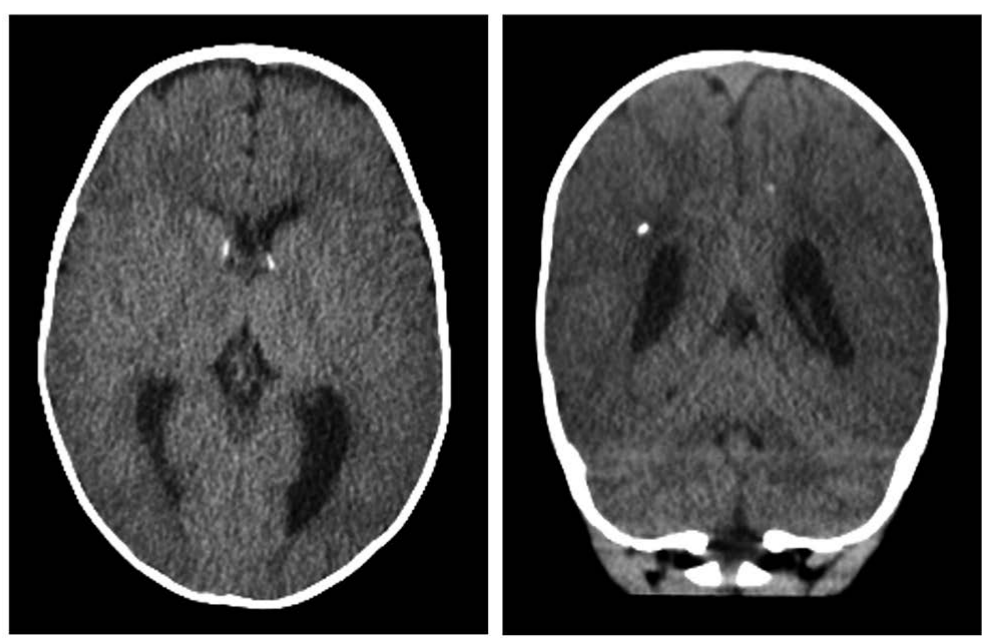

Figura 2. A. TC de cerebro sin contraste gemela 1: imágenes focales puntiformes con densidad cálcica de distribución supratentorial subcortical y periventricular. B. TC de cerebro sin contraste gemela 2: ventriculomegalia, imágenes puntiformes con densidad cálcica a nivel de los centros semiovales, corona radiata, núcleos caudados y periventriculares.
Recibieron tratamiento con pirimetamina (P) $1 \mathrm{mg} /$ $\mathrm{kg} / \mathrm{dosis}$ cada $24 \mathrm{~h}$ vía oral (dosis de ataque de $2 \mathrm{mg} / \mathrm{kg}$ / dosis cada $24 \mathrm{~h}$ los primeros dos días de tratamiento), sulfadiazina (S) $50 \mathrm{mg} / \mathrm{kg} /$ dosis cada $12 \mathrm{~h}$ vía oral, metilprednisona $1 \mathrm{mg} / \mathrm{kg}$ /día cada $8 \mathrm{~h}$ vía oral (por coriorretinitis activa) y ácido folínico (AF) $10 \mathrm{mg}$ tres veces por semana por vía oral durante el primer año de vida. A partir de los seis meses de edad se utilizó la pauta de tratamiento con pirimetamina $1 \mathrm{mg} / \mathrm{kg} /$ día, tres veces por semana. Durante el tratamiento se hicieron controles con hemograma, función renal y hepática mensual hasta la finalización del mismo, no presentando alteraciones que requirieran la suspensión de la terapia.

En la actualidad, han completado dos años de seguimiento multidisciplinario, recibiendo estimulación temprana con controles oftalmológicos, neurológicos, auditivos y clínicos periódicos no habiéndose detectado reactivación de lesiones oculares, ni progresión de compromiso neurológico.

\section{Discusión}

La toxoplasmosis aguda en la mujer embarazada, por lo general, es asintomática o subclínica y se detecta por métodos serológicos. La transmisión del parásito al feto ocurre en la primoinfección durante el embarazo y aumenta gradualmente, estimándose en promedio $15 \%$ en el primer trimestre, $44 \%$ en el segundo y $71 \%$ en el último trimestre del embarazo. Por otro lado, la gravedad del compromiso fetal es mayor durante el primer trimestre de gestación con aborto espontáneo o compromiso neurológico grave, mientras que la infección adquirida al final del embarazo puede dar un RN asintomático ${ }^{1,3}$.

En Argentina, se recomienda el estudio serológico de rutina de la mujer embarazada para ofrecer tratamiento oportuno y reducir la transmisión vertical y/o el daño 
fetal. El cribado universal debería iniciarse antes de las 12 semanas de gestación con $\mathrm{IgG}$ por inmunofluorescencia indirecta (IFI), enzimoinmunoensayo (EIA), reacción de Sabin Feldman (SF) o aglutinación directa (AD), métodos con buena sensibilidad y especificidad. La aparición de los anticuerpos en la infección aguda tiene una curva de ascenso rápida, con títulos máximos entre las 6 a 12 semanas. Las diferentes técnicas de detección de anticuerpos tienen sensibilidad, especificidad y tiempo de aparición variable ${ }^{7}$.

Si la IgG es negativa, debe repetirse la serología en cada trimestre y brindarse consejería para evitar la primoinfección. Estas recomendaciones idealmente deben ser dadas por escrito y referidas especialmente al adecuado lavado de manos previo a la ingesta de alimentos y al cocinar, consumo de carne bien cocida, limpieza de los utensilios que tuvieron contacto con carne cruda, evitar la ingesta de vegetales crudos si no se puede asegurar la adecuada higiene de los mismos, uso de guantes al hacer trabajos de jardinería, evitar el contacto con excretas de gatos, entre otras?

Si la IgG es positiva debe realizarse la detección de IgM. Esta inmunoglobulina se encuentra presente en la infección aguda y ausente en la forma crónica, aunque puede persistir reactiva durante meses o años. Su detección en el primer trimestre podría sugerir una infección aguda, pero no indica con seguridad que la infección se haya producido durante la gestación. Por lo tanto, la sola determinación de IgM no es suficiente para diagnosticar ni descartar una toxoplasmosis aguda.

Los resultados positivos de las pruebas de cribado requieren de reacciones más complejas confirmatorias, tales como la reacción de SF, IFI, inmunocaptura y prueba de avidez. En nuestro medio, la prueba de avidez es la más utilizada. Se basa en la baja avidez de los anticuerpos tipo IgG por los antígenos parasitarios en los primeros tres a cinco meses de la infección, por lo que una prueba de alta avidez en el primer trimestre de embarazo permite descartar una infección reciente. Sin embargo, aunque una baja avidez sugiere una infección aguda, esta no es confirmatoria y debe ser interpretada en el contexto de las demás pruebas realizadas?

Ante la presencia de un perfil serológico compatible con una infección aguda se sugiere realizar una amniocentesis a partir de las 18-20 semanas de gestación y al menos pasadas seis semanas desde la infección, para la detección de $T$. gondii por RPC en el líquido amniótico. Esta técnica no está estandarizada, tiene resultados falsos positivos y negativos con una sensibilidad de $65-90 \%$ y una especificidad cercana a $100 \%{ }^{3,7}$. La metodología diagnóstica empleada en distintos reportes o series de casos varía según la disponibilidad en los distintos centros. Por ejemplo, en algunos casos el diagnóstico de infección materna se hizo por detección de IgM y seroconversión de
IgG por EIA 6 , en otros se emplearon muestras pareadas de IgG separadas por al menos una semana ${ }^{8}$ y en otros, la confirmación diagnóstica se hizo por métodos directos con la detección del parásito por RPC en el líquido amniótico $^{8}$. En el caso que presentamos, el diagnóstico de la infección aguda en nuestra paciente embarazada se hizo por la seroconversión de IgG e IgM por técnica de quimioluminiscencia a las 33 semanas de gestación y la baja avidez de la IgG. No se realizó amniocentesis debido a que no se encuentra disponible en nuestro centro.

En mujeres embarazadas con diagnóstico probable o confirmado de toxoplasmosis está indicado realizar el seguimiento ecográfico para detectar anomalías fetales. La ventriculomegalia y las calcificaciones intracraneales son las lesiones más observadas. Las lesiones extracraneales son menos frecuentes e incluyen ascitis, hidropesía, hepatoesplenomegalia y engrosamiento placentario ${ }^{3}$. En el caso que presentamos, la ecografía fue normal, sin hallazgos sugerentes de compromiso fetal o placentario.

Después del parto, el RN debe ser evaluado clínicamente y mediante determinaciones de $\operatorname{IgG}, \operatorname{IgM}$ e IgA. La IgG atraviesa la placenta y el título obtenido al nacimiento generalmente coincide con el materno. Títulos de IgG significativamente mayores o la presencia de IgM y/o IgA, que no atraviesan la placenta, son un indicio de infección prenatal. Para la detección de $\operatorname{IgM}$ e $\operatorname{IgA}$ las técnicas de inmunocaptura son las más adecuadas por su sensibilidad y especificidad. La técnica de immunosorbent agglutination assay (ISAGA) para la detección de IgM tiene una sensibilidad de $80 \%$ y para $\operatorname{IgA}$ de $83 \%$, con la realización de ambas determinaciones la sensibilidad llega $92 \%{ }^{3,7}$

La detección directa del parásito puede realizarse con RPC en sangre, LCR y orina como complemento de la serología, en caso de no detectarse IgM e IgA específicas. Permite la detección de $\mathrm{ADN}$ de parásitos vivos y muertos, con una sensibilidad de $60-80 \%$ y una especificidad de casi $100 \%$. Su utilidad es limitada en sangre debido a la corta parasitemia. La sensibilidad en el cordón umbilical y en la placenta es de alrededor de 16 y 50-87\%; respectivamente. Es una técnica aún no estandarizada y poco utilizada ${ }^{1,3}$. En diferentes reportes, el diagnóstico de infección congénita se realizó por determinación de IgM por ISAGA ${ }^{6}$ y ELISA ${ }^{9}$ en RN sintomáticos. En uno de los casos, se empleó también RPC en sangre para detectar el parásito ${ }^{6}$. En el caso que presentamos, ambas gemelas tuvieron serología reactiva para toxoplasmosis IgG e IgM por método de quimioluminiscencia. El resultado de IgM reactiva en sangre con un índice de 3 confirmó la infección congénita. No pudimos realizar estudios microbiológicos moleculares específicos como la detección del parásito mediante RPC en sangre, LCR ni orina por no estar disponible en nuestro centro.

Para determinar la extensión del compromiso del RN, 
es necesario realizar un hemograma, estudio de la función hepática, fondo de ojos e imagen del sistema nervioso central (SNC). La ecografía permite detectar lesiones de mayor tamaño en el parénquima cerebral mientras que la RM tiene mayor sensibilidad que la TC al permitir la detección de microcalcificaciones no visibles. También se recomienda realizar una punción lumbar con estudio citoquímico del LCR donde puede detectarse un aumento del nivel de proteínas ${ }^{7}$. En los casos que presentamos, no se observó compromiso hematológico ni hepático. El fondo de ojos mostró una coriorretinitis activa bilateral en ambas gemelas y la ecografía cerebral constató la presencia de calcificaciones en el parénquima cerebral.

En estudios que comparaban el compromiso en embarazos monocigóticos y dicigóticos, se detectó que, en los primeros, ambos gemelos presentaban manifestaciones clínicas mientras que, en los segundos, uno de los RN podía ser completamente asintomático ${ }^{4-6,10-12}$. En una serie de Argentina, 79\% de los RN con toxoplasmosis congénita presentaron coriorretinitis, calcificaciones cerebrales y compromiso macular con frecuencia variable ${ }^{10}$. En nuestro caso, ambas gemelas tuvieron igual compromiso (oftalmológico y cerebral) lo cual coincide con lo descrito en embarazos monocigóticos monocoriales.

La elección del tratamiento de la mujer embarazada con toxoplasmosis aguda depende del momento de diagnóstico y de los resultados de la amniocentesis, en caso de realizarse. Si la infección se diagnostica en el primer trimestre se recomienda iniciar con espiramicina 1 gramo cada 8 h vía oral debido a los efectos teratógenos de pirimetamina. Puede rotarse al esquema de $\mathrm{P}+\mathrm{S}+\mathrm{AF}$ a partir de las 18 semanas de gestación 7 . Asimismo, si la probabilidad de compromiso fetal es alta (ecografía fetal patológica, RPC positiva en líquido amniótico) se sugiere el tratamiento combinado $\mathrm{P}+\mathrm{S}+\mathrm{AF}$. Si bien, no se ha demostrado superioridad de un esquema sobre el otro, se conoce que la espiramicina alcanza en el feto alrededor de $50 \%$ de los niveles plasmáticos maternos. En caso de detectarse compromiso neurológico podría emplearse para el tratamiento debido a que podría tener algún nivel de pasaje a través de la barrera hematoencefálica $(\mathrm{BHE})^{3}$. En el caso que presentamos, el tratamiento se inició por la seroconversión de títulos maternos. Al no presentar ecografías prenatales patológicas ni contar con muestra de líquido amniótico confirmatoria de infección, se completó con espiramicina hasta el momento del parto.

El tratamiento del $\mathrm{RN}$ sintomático consiste en la administración de P $1 \mathrm{mg} / \mathrm{kg} /$ dosis cada 24 h, S $50 \mathrm{mg} /$ $\mathrm{kg} /$ dosis cada $12 \mathrm{~h}$ y AF $10 \mathrm{mg}$ trisemanal durante los primeros 12 meses de vida según las recomendaciones actuales $^{3,7}$. En los diferentes casos publicados se emplearon esquemas de P, S, AF hasta los 12 a 15 meses de edad ${ }^{6,7}$. En caso de una coriorretinitis activa o de proteinorraquia $>1 \mathrm{~g} / \mathrm{dL}$ en LCR se agregan corticoesteroides hasta su normalización ${ }^{3,7}$. En estas gemelas se inició tratamiento con los tres fármacos y se agregó corticoesteroides por la presencia de una coriorretinitis activa.

Los eventos adversos más frecuentes asociados al tratamiento son hematológicos, con anemia macrocítica, neutropenia y trombocitopenia. En un estudio realizado en Brasil, 44\% de los niños que se encontraban recibiendo $\mathrm{P}, \mathrm{S}$ y AF tuvieron algún evento adverso hematológico. La neutropenia se presentó en 33\% de los casos y revirtió con el aumento de la dosis de AF o con la suspensión transitoria del tratamiento ${ }^{13}$. En nuestro caso, se hizo control con hemograma mensual durante todo el tratamiento y no se detectó la presencia de compromiso de series celulares que requirieran la suspensión del tratamiento.

El seguimiento debe ser multidisciplinario y frecuente para la detección precoz de reactivaciones o de aparición de compromiso orgánico. Es fundamental el seguimiento con fondo de ojos para detectar en forma temprana posibles reactivaciones. Debe realizarse con una frecuencia trimestral durante el primer año y medio de vida y luego una o dos veces al año hasta que el niño pueda referir cambios en la visión. Una etapa del desarrollo en la cual pueden producirse reactivaciones es la pubertad por lo que el seguimiento debe hacerse cada seis meses en este momento. La sordera neurosensorial es frecuente, por lo que debe realizarse una evaluación inicial con potenciales evocados auditivos al nacimiento y al año de edad y anualmente hasta que el niño pueda referir cambios auditivos. Respecto al seguimiento neurológico, en cada control clínico debe evaluarse la adquisición de pautas madurativas y el perímetro cefálico. Si se detectan alteraciones deberá realizarse una RM de cerebro y evaluación especializada ${ }^{3}$. Las pacientes que presentamos, actualmente han completado dos años de seguimiento multidisciplinario y estimulación temprana. Hasta el momento no se han detectado signos de reactivación de la coriorretinitis en los controles oftalmológicos trimestrales ni aparición de nuevas lesiones ni progresión de las ya existentes.

\section{Conclusiones}

La toxoplasmosis congénita en gemelos es una situación muy poco frecuente. No presenta diferencias en cuanto al diagnóstico y el tratamiento en embarazos únicos. La gravedad del cuadro es mayor si la infección se adquiere al inicio de la gestación y disminuye a medida que el embarazo avanza. El compromiso de los RN en embarazos gemelares es variable según sea monocigótico o dicigótico. Es fundamental el seguimiento multidisciplinario durante la infancia para la detección precoz de reactivaciones o de la aparición o progresión de lesiones que generen secuelas e incapacidad, brindando especial atención al compromiso ocular. 


\section{Caso Clínico}

\section{Referencias bibliográficas}

1.- Peyron F, Wallon M, Kieffer F. Capítulo 31. Toxoplasmosis. Remington and Klein's Infectious Diseases of the Fetus and Newborn Infant. $8^{\circ}$ Edición Saunders 2015. Pág 9491042 .

2.- Torgerson P R, Mastroiacovo P. The global burden of congenital toxoplasmosis: a systematic review. Bull World Health Organ 2013; 91: 501-8. doi: 10.2471/BLT.12.111732.

3.- Baquero-Artigao F, del Castillo Martín F, Fuentes Corripio I, Goncé Mellgren A, Fortuny Guasch C, Fernández-Miranda M. de la Calle, et al. Guía de la Sociedad Española de Infectología Pediátrica para el diagnóstico y tratamiento de la toxoplasmosis congénita. An Pediatr (Barc) 2013; 79: e1-116. doi: 10.1016/ j.anpedi.2012.12.001.

4.- Bischoff A R, Friedrich L, Cattan J M, Uberti F A. Incidence of symptomatic congenital toxoplasmosis during ten year in a brazilian hospital. Pediatr Infect Dis J 2016; 35:1313-6. doi: 10.1097/ INF.0000000000001307.
5.- Thapa R, Banerjee P, Akhtar N, Jain T. Discordance for congenital toxoplasmosis in twins. Indian J Pediatr 2009; 76: 1069- 70. doi: 10.1007/s12098-009-0208-9.

6.- Peyron F, Ateba A B, Wallon M, Kodjikian L, Binquet C, Fleury J, et al. Congenital toxoplasmosis in twins: a report of fourteen consecutive cases and a comparison with published data. Pediatr Infect Dis J 2003; 22: 695-701. doi: 10.1097/01. inf.0000078354.15385.76.

7.- Durlach R, Kaufer F, Carral L, Freuler C, Cariotto M, Rodríguez M, et al. Consenso argentino de toxoplasmosis congénita. Medicina (B Aires) 2008; 68: 75-87.

8.- Mc Crossin B, Roberton N. Congenital skin defects, twins and toxoplasmosis. J Royal Soc Med 1989; 82: 108-9. doi: 10.1177/014107688908200219.

9.- Benjamin B, Brickman H, Neaga A. Congenital toxoplasmosis in twins. Canad M A J 1959; 80: 639-43.

10.- Carral L, Kaufer F, Pardini L, Durlach R, Moré G, Venturini M, et al. Toxoplasmosis congénita: diagnóstico serológico, RPC, aislamiento y caracterización molecular de Toxoplasma gondii. Rev Chilena Infectol 2018; 35: 36-40. doi: 10.4067/s071610182018000100036 .

11.- Tjalma W, Vanderheyden T, Naessens A, Vanderheyden J, Foulon W. Discordant prenatal diagnosis of congenital toxoplasmosis in a dizygotic pregnancy. Eur J Obstet Gynecol Reprod Biol 1998; 79: 107-8. doi:10.1016/ s0301-2115(98)00047-5.

12.- Morais Falavigna D L, Roncada E V, Nakazora D, Castilho Pelloso M, Morais Falavigna L F, Marques de Araújo S, et al. Congenital toxoplasmosis in dizygotic twins, Paraná, Brazil. Case report. Rev Inst Med Trop Sao Paulo 2007; 49: 117-8. doi:10.1590/S0036-46652007000200010.

13.- Carellos E, de Andrade J, Romanelli R, Tibúrcio J, Januário J, Vasconcelos-Santos D, et al. High frequency of bone marrow depression during congenital toxoplasmosis therapy in a cohort of children identified by neonatal screening in Minas Gerais, Brazil. Pediatr Infect Dis J 2017; 36: 1169-76. doi: 10.1097/INF.0000000000001561. 